\title{
Reflexo da Saúde Mental dos profissionais atuantes no Covid-19, no interior do Pará
}

\section{Reflection of the Mental Health of professionals working at Covid-19, in the interior of Pará}

\author{
Pamela Cássia Gomes Silva ${ }^{1 *}$, Nathalia Menezes Dias ${ }^{2}$
}

\begin{abstract}
RESUMO
Objetivo: Analisar a saúde mental dos profissionais atuantes na epidemia do covid-19 no Hospital Regional de Tucuruí (HRT) e Unidade de Pronto Atendimento (UPA) no município de Tucuruí no estado do Pará. Métodos: A metodologia da pesquisa trata-se de um estudo descritivo, transversal e quantitativo. A amostra correspondeu a 61 da totalidade dos profissionais médicos, enfermeiros, técnicos de enfermagem e fisioterapeutas. Os dados analisados são referentes ao período de agosto de 2020 a julho de 2021. Resultados: Diante desse fato faz se necessário desenvolver estratégias de apoio psicológico a esses profissionais para promover a saúde mental, utilizando tecnologias online como forma de distanciamento físico. Conclusão: O cenário de pandemia acarretou alteração da saúde mental não apenas da classe dos profissionais de saúde. As medidas restritivas impostas tais como: distanciamento, cuidados de higiene, uso constante de EPI, transmissibilidade e infecção, aliado a falta de capacitação e reciclagem em relação ao Covid-19, e aumento na jornada de trabalho ocasionando sintomas de depressão, ansiedade, irritabilidade, insônia ao serem somatizadas podem agravar negativamente para doença mental.
\end{abstract}

Palavras-chave: Saúde mental; Coronavírus; Intervenção psicossocial.

\begin{abstract}
Objective: To analyze the mental health of professionals working in the covid-19 epidemic at the Regional Hospital of Tucuruí (HRT) and Emergency Care Unit (UPA) in the municipality of Tucuruí in the state of Pará. Methods: The research methodology is about a descriptive, cross-sectional and quantitative study. The sample corresponded to 61 of all medical professionals, nurses, nursing technicians and physiotherapists. The analyzed data refer to the period from August 2020 to July 2021. Results: Given this fact, it is necessary to develop psychological support strategies for these professionals to promote mental health, using online technologies as a form of physical distance. Conclusion: The restrictive measures imposed such as: distancing, hygiene care, constant use of PPE, transmissibility and infection, combined with a lack of training and recycling in relation to Covid-19, and an increase in working hours, causing symptoms of depression and anxiety irritability, insomnia when somatized can negatively aggravate mental illness.
\end{abstract}

Keywords: Mental health; Coronaviruses; Psychosocial intervention.

\footnotetext{
${ }^{1}$ Faculdade de Medicina e Odontologia São Leopoldo Mandic (SLMANDIC). *E-mail: pamelacassia@hotmail.com

${ }^{2}$ Universidade do Estado do Pará (UEPA)
} 


\section{INTRODUÇÃO}

Segundo dados da Organização Mundial de Saúde (OMS), o surto da COVID-19, como se chamou posteriormente a doença respiratória causada pelo vírus SARS-CoV-2, iniciou-se na China em dezembro de 2019. Desde então, tem se alastrado por diversos locais e populações do mundo. Os primeiros contágios pelo vírus apareceram na China e seguiram por países como Japão, França, Espanha, Itália e Reino Unido. A pandemia Dados oficiais do Brasil, de 10 de julho de 2021, registram 19.069.003 casos confirmados de COVID-19, sendo 532.893 óbitos, com indicadores de 2,8\% de taxa de letalidade. Em função desses números o Brasil assumiu o $2^{\circ}$ lugar no ranking mundial de casos confirmados e óbitos relacionados a pandemia, perdendo apenas para os Estados unidos, segundo a World Health Organização (OMS) (OPAS/OMS, 2020; DUARTE et al., 2020).

Embora a região norte tenha sofrido o acréscimo exponencial em relação ao Covid-19, durante a primeira onda, dentre as regiões brasileiras, hoje o cenário atual distinto apresenta decréscimo relacionados aos números de casos e óbitos provocados pelo Covid-19 (BRASIL, 2020a).

O SARS-CoV-2 é altamente transmissível por gotículas e contato, principalmente em locais fechados e ambientes hospitalares. Um indivíduo com infecção pelo novo Corona vírus transmite para outras duas ou três pessoas, dependendo das condições ambientais. Como consequência desse fato a pandemia trouxe histeria e paranoia para o público em geral, ocasionando surtos; pois o medo é comum e pode levar a um comportamento que oscila podendo atingir a todos e para a Covid-19 isso é ainda mais perceptível uma vez que pouco se sabe e muito se especula. Logo o ritmo de disseminação é muito rápido e não há tratamento específico (BRASIL, 2020a).

Diante da inexatidão do Covid-19, pode-se perceber que a equipe de saúde apresentou níveis elevados de ansiedade e depressão, isso porque estando mais expostos correm mais riscos e sentem medo por si e pelos familiares. Falta de apoio de comunicação e de treinamento são fatores de risco que aumentam a possibilidade do desenvolvimento de doenças psicológicas. Durante as pandemias o foco está na doença em si e não nos transtornos mentais que dela possam advir. Entretanto, a Covid-19 trouxe a necessidade de se pensar sobre as questões de saúde mental, uma vez que se observou a crescente sobrecarga desta (BARBOSA et al., 2020; PRADO et al., 2020).

Em linhas gerais, na vigência de pandemias, a saúde física das pessoas e o combate ao agente patogênico são os focos primários de atenção de gestores e profissionais da 
saúde, de modo que as implicações sobre a saúde mental tendem a ser negligenciadas ou subestimadas (OLIVEIRA et al., 2020). Contudo, medidas adotadas para reduzir as implicações psicológicas da pandemia não podem ser desprezadas neste momento (SCHMIDT et al., 2020).

O objetivo do estudo é avaliar os impactos negativos da saúde mental aos profissionais atuantes na linha de frente da pandemia do COVID-19 no Município de Tucuruí.

\section{MÉTODO}

A metodologia da pesquisa trata-se de um estudo descritivo, transversal e quantitativo. O estudo foi realizado no município de Tucuruí no estado do Pará, com os profissionais da linha enfrentamento da Covid-19 no município, com o intuito de avaliar qual foi o impacto da pandemia na saúde Mental deles. Os dados analisados são referentes ao período de agosto de 2020 a julho de 2021. O instrumento de coleta de dados foi um questionário online, ao qual foi possibilitou uma avaliação descritiva e epidemiológica dessa população, com os fatores potencializadores estressantes relacionados ao exercício da profissão durante a pandemia. A pesquisa foi submetida ao Comitê de Ética e Pesquisa (CEP) com número do CAAE: 35616720.8.0000.5374.

A amostra da pesquisa foi realizada com profissionais do Hospital Regional de Tucuruí (HRT), sendo dividido em dois setores: uma Ala do Covid-19 e uma Ala de UTI Covid-19, e com os profissionais da Unidade Pronto Atendimento de Tucuruí (UPA) Covid-19. Foram convidados a participar do estudo todos os profissionais que atendiam aos critérios de inclusão, a saber: prestar serviço na ala de Covid-19 do HRT e UPA, setor Covid-19.

A amostra obtida correspondeu a 61 profissionais de saúde dentre estes médicos, enfermeiros, técnicos de enfermagem e fisioterapeutas. Foram devidamente esclarecidos em relação aos objetivos da pesquisa, principalmente no que diz respeito à confiabilidade e não identificação dos envolvidos, além dos benefícios que poderão ser alcançados; eles poderiam, inclusive, se recusar a participar do estudo ou sair. Após autorização do termo de consentimento livre e informado, responderam ao questionário.

A análise da pesquisa sobre os impactos da Covid-19 na saúde mental desses profissionais se faz necessárias para informar as decisões políticas, esforços de prevenção e programas de apoio a esses profissionais para diminuir ou reduzir os transtornos 
ocasionados pela pandemia, com a finalidade de reabilitá-los aos seus respectivos quadros funcionais.

\section{RESULTADOS}

Os dados coletados de 61 profissionais de saúde da linha de frente do enfrentamento do Covid-19, são pertencentes as categorias de: Médicos 6 (10\%), Enfermeiros 17 (28,3\%), Técnicos de Enfermagem 35 (58,3\%) e Fisioterapeutas 3 $(3,3 \%)$, cuja a idade predominante foi entre 30 a 40 anos 30 (50\%), com predomínio do gênero feminino 39 (65\%), com formação profissional variam de 1 a 5 anos. A maioria dos participantes $33(55 \%)$ relatou ser casados e terem religião, e não se consideram pertences ao grupo de risco para Covid-19.

Tabela 1 - Características dos profissionais incluídos nos estudos. Tucuruí, Brasil, 2021.

\begin{tabular}{|c|c|c|c|}
\hline Variáveis & & $\mathrm{N}$ & $\%$ \\
\hline Gênero & $\begin{array}{l}\text { Masculino } \\
\text { Feminino }\end{array}$ & $\begin{array}{l}21 \\
39\end{array}$ & $\begin{array}{l}35 \% \\
65 \%\end{array}$ \\
\hline Idade & $\begin{array}{c}20 \text { a } 30 \text { anos } \\
30 \text { a } 40 \text { anos } \\
40 \text { a } 50 \text { anos } \\
\text { Acima de } 50 \text { anos }\end{array}$ & $\begin{array}{c}17 \\
30 \\
11 \\
2\end{array}$ & $\begin{array}{c}28,3 \% \\
50 \% \\
18,3 \% \\
3,3 \%\end{array}$ \\
\hline Religião & $\begin{array}{l}\text { Sim } \\
\text { Não }\end{array}$ & $\begin{array}{c}51 \\
9\end{array}$ & $\begin{array}{l}85 \% \\
15 \%\end{array}$ \\
\hline Função & $\begin{array}{c}\text { Médico } \\
\text { Enfermeiro (a) } \\
\text { Téc. de Enfermagem } \\
\text { Fisioterapeuta }\end{array}$ & $\begin{array}{c}6 \\
17 \\
35 \\
2\end{array}$ & $\begin{array}{c}10 \% \\
28,3 \% \\
58,3 \% \\
3,3 \%\end{array}$ \\
\hline $\begin{array}{c}\text { Tempo de Formação } \\
\text { Profissional }\end{array}$ & $\begin{array}{c}1 \text { a } 5 \text { anos } \\
5 \text { a } 10 \text { anos } \\
10 \text { a } 15 \text { anos } \\
15 \text { a } 20 \text { anos } \\
\text { Acima de } 20 \text { anos }\end{array}$ & $\begin{array}{c}25 \\
12 \\
11 \\
8 \\
4\end{array}$ & $\begin{array}{c}41,7 \% \\
20 \% \\
18,3 \% \\
13,3 \% \\
6,7 \%\end{array}$ \\
\hline Casados & $\begin{array}{l}\text { Sim } \\
\text { Não }\end{array}$ & $\begin{array}{l}33 \\
27\end{array}$ & $\begin{array}{l}55 \% \\
45 \%\end{array}$ \\
\hline Grupo de Risco & $\begin{array}{l}\text { Sim } \\
\text { Não }\end{array}$ & $\begin{array}{l}17 \\
43\end{array}$ & $\begin{array}{l}28,3 \% \\
71,7 \%\end{array}$ \\
\hline
\end{tabular}

Em relação a rotina de trabalho $45(75 \%)$ dos profissionais verificaram alterações em suas rotinas de trabalho; dentre esses profissionais 43 (72,9\%) observaram alterações emocionais. Dentre as alterações: depressão, ansiedade, insônia e irritabilidade, os 
profissionais $22(38,6 \%)$ relataram ansiedade e $12(21,1 \%)$ unânimes em ter apresentado todos esses sentimentos. Embora $55(91,7 \%)$ dos profissionais não possuírem doença mental ou física antes da pandemia e $27(47,4 \%)$ relataram assistir filmes e séries como forma de aliviar a tensão (Tabela 2).

Em relação aos determinantes de enfrentamento do Covid-19 com capacitação, treinamento e prevenção, 35 (58,3\%) dos profissionais não foram habilitados e preparados durante a pandemia e ainda $39(68,4 \%)$ tiveram aumento na carga horária de trabalho por substituir colegas que adoeceram. Sendo que $46(76,7 \%)$ pensaram em abandonar a profissão (Tabela 2).

Tabela 2 - Fatores associados a transtornos mentais comuns nos profissionais, segundo características do Covid-19 nos estudos. Tucuruí, Brasil, 2021.

Variáveis

\begin{tabular}{|ccc|}
\hline & N & $\%$ \\
Sim & 45 & $75 \%$ \\
Não & 43 & $15 \%$ \\
Talvez & 6 & $10 \%$ \\
Sim & 43 & $72,9 \%$ \\
Não & 7 & $11,9 \%$ \\
Talvez & 9 & $15,3 \%$ \\
Depressão & 0 & $28,1 \%$ \\
Insônia & 16 & $12,3 \%$ \\
Irritabilidade & 7 & $38,6 \%$ \\
Ansiedade & 22 & $21,1 \%$ \\
Todas as respostas & 12 & \\
Sim & 5 & $8,3 \%$ \\
Não & 55 & $91,7 \%$ \\
Atividade Física & 19 & $33,3 \%$ \\
Filmes e séries & 27 & $47,4 \%$ \\
Bebidas alcoólicas & 9 & $15,8 \%$ \\
Cigarros e afins & 2 & $3,5 \%$ \\
Sim & 25 & $41,7 \%$ \\
Não & 35 & $58,3 \%$ \\
& & \\
Sim & 39 & $68,4 \%$ \\
Não & 18 & $31,6 \%$ \\
Sim & & $23,3 \%$ \\
Não & 14 & $76,7 \%$ \\
& 46 &
\end{tabular}

Rotina de Trabalho

Alteração Emocional

Alterações Emocionais

Doença Mental

Hábitos alívio de tensão

Capacitação Profissional

Aumento Carga Horária

Abandonar a Profissão

Fonte:

Durante a atuação profissional e assistência aos pacientes contaminados pelo Covid-19, 42 (70\%) dos profissionais não se consideram bem remunerados, e 53 (88,3\%) estão preocupados em relação a escassez dos EPIs, e a maioria dos profissionais 31 
$(52,5 \%)$ relataram tensões em ambos os pacientes, sejam na Ala do Covid-19 ou pacientes intubados e dentre as suas maiores preocupações estão o medo e receio desse profissionais infectar outras pessoas 30 (49,18\%), seguidos de se infectarem por Covid-19 28 (45,90\%), infectarem seus familiares 22 (36,06\%) e morrer de Covid19 19 (31,14\%).

Em relação a rotina de trabalho $20(33,3 \%)$ dos profissionais não realizaram teste rápido preventivamente; porém ao serem infectados no ambiente de trabalho $13(39,4 \%)$ realizaram teste de rápido de Covid-19, $2(6,1 \%)$ já realizaram o protocolo imediata de medicação, 5 (15,2\%) foram afastados de suas atividades diárias, e 13 (39,4\%) desses profissionais receberam todos os procedimentos descritos anteriormente, e descreveram os seguintes sintomas: $9(27,3 \%)$ perda de paladar, $5(15,2 \%)$ perda de paladar, $2(6,1 \%)$ diarreia, $9(27,3 \%)$ dor muscular e 8 (24,2\%) desconforto respiratório ( Tabela 3). Apesar dos dados citados e encontrados os profissionais foram unanime ao relatarem que atuariam no Covid-19 ou em outra pandemia (Tabela 3).

Tabela 3 - Características ocasionadas pelo Covid-19 nos estudos. Tucuruí, Brasil, 2021.

Variáveis

\begin{tabular}{|c|c|c|c|}
\hline Variáveis & & $\mathrm{N}$ & $\%$ \\
\hline Remuneração & $\begin{array}{c}\text { Sim } \\
\text { Não } \\
\text { Talvez }\end{array}$ & $\begin{array}{c}6 \\
42 \\
42\end{array}$ & $\begin{array}{l}10 \% \\
70 \% \\
20 \%\end{array}$ \\
\hline Escassez EPI & $\begin{array}{l}\text { Sim } \\
\text { Não }\end{array}$ & $\begin{array}{c}53 \\
7\end{array}$ & $\begin{array}{l}88,3 \% \\
11,7 \%\end{array}$ \\
\hline Tensões na Rotina de trabalho & $\begin{array}{l}\text { Isolamento de Covid-19 } \\
\text { Pacientes entubados } \\
\text { Ambos os pacientes }\end{array}$ & $\begin{array}{c}3 \\
25 \\
31\end{array}$ & $\begin{array}{c}5,1 \% \\
42,4 \% \\
52,5 \%\end{array}$ \\
\hline Momento de maior tensão & $\begin{array}{c}\text { Infectar pessoas Covid19 } \\
\text { Infectar-se por Covid19 } \\
\text { Infectar familiar } \\
\text { Morrer }\end{array}$ & $\begin{array}{l}30 \\
28 \\
22 \\
19\end{array}$ & $\begin{array}{l}49,18 \% \\
45,90 \% \\
36,06 \% \\
31,14 \%\end{array}$ \\
\hline Teste Rápido & $\begin{array}{l}\text { Sim } \\
\text { Não }\end{array}$ & $\begin{array}{l}20 \\
40\end{array}$ & $\begin{array}{l}33,3 \% \\
66,7 \%\end{array}$ \\
\hline Contaminação por Covid19 & $\begin{array}{c}\text { Teste de Covid19 } \\
\text { Protocolo medicamentoso } \\
\text { Afastado da rotina } \\
\text { Todos os procedimentos }\end{array}$ & $\begin{array}{c}13 \\
2 \\
5 \\
13\end{array}$ & $\begin{array}{c}39,4 \% \\
6,1 \% \\
15,2 \% \\
39,4 \%\end{array}$ \\
\hline Sintomas observados & $\begin{array}{c}\text { Perda de Paladar } \\
\text { Perda de Olfato } \\
\text { Diarreia } \\
\text { Dor muscular } \\
\text { Desconforto respiratório }\end{array}$ & $\begin{array}{l}9 \\
5 \\
2 \\
9 \\
8\end{array}$ & $\begin{array}{c}27,3 \% \\
15,2 \% \\
6,1 \% \\
27,3 \% \\
24,2 \%\end{array}$ \\
\hline
\end{tabular}

\section{DISCUSSÃO}


A Pandemia do Covid-19 tornou-se uma emergência em saúde pública internacional, em função das inúmeras vítimas dispersas em escala mundial, colapsando os distintos sistemas de saúde, tal problemática desenvolveu distintos aspectos psicológico e metais aos profissionais de saúde ao longo da Pandemia (BARBOSA et al., 2020; PRADO et al., 2020; SILVA et al., 2020).

Estudos chineses realizados desde o início da pandemia confirmam os impactos psicológicos na população tais como: ansiedade, percepção de estresse e depressão, com aumento gradual no transcorrer da doença (BARBOSA et al., 2020; PRADO et al., 2020; OLIVEIRA et al., 2020; SILVA et al., 2020). Tais alterações emocionais foram verificadas e potencializadas pela população de maneira geral, nos profissionais de saúde são exageradas e acrescidas de variáveis que geram dúvidas; pela alteração da rotina de trabalho, pela compreensão da complexidade da doença seja em relação aos insumos, prevalência e contaminação da doença, com aumento da carga horária, já que os mesmos prestam assistência na linha de frente do enfrentamento do Covid-19.

O profissionais de saúde de todo o país com síndrome gripal inicial e depois confirmados e positivados para a Covid-19, segundo o boletim epidemiológico do Ministério de saúde foram distribuídos em ordem decrescente: técnicos de enfermagem, seguidos de enfermeiro e medicos (BRASIL, 2020a), corroborando com o estudo ao qual foi possível verificar maior quantitativo de técnicos de enfermagem, seguidos pelos enfermeiros ocorrendo maior probabilidade de contaminação desses profissionais por rotatividade e equipe; pois estão mais expostos durante a assistência e prescrição medicamentosa aos pacientes.

Os profissionais japoneses de saúde atuantes no combate ao enfrentamento do Covid-19; após o surto de SARS, relataram aumento no consumo de álcool, Tabaco (SHIGEMURA et al., 2020). Os profissionais de saúde do Município de Tucuruí adquiriram hábitos como alívio de tensão tais como: atividade física, filmes, séries, seguidos por bebidas alcóolicas, cigarros e afins.

Embora existam pesquisas em relação aos impactos na Saúde Mental dos profissionais de saúde neste período de pandemia, onde $25 \%$ médicos residentes em Brasília, cogitaram troca de especialidade (BRASIL, 2020b). Em contrapartida a maioria dos profissionais do município de Tucuruí embora tenha percebido alterações emocionais, na rotina de trabalho, não tenham realizado capacitação profissional, e nem remuneração adequada, tais fatores prejudiciais não os fizeram pensar em abandonar a profissão. 


\section{CONCLUSÃO}

A pandemia do Covid-19 desencadeou uma crise na saúde dos profissionais, diante deste cenário cabe as instituições hospitalares, bem como os governos Federal e Estadual traçar estratégias a fim de minimizar os impactos psicossocial ocasionados aos profissionais na linha de frente do enfrentamento do Covid-19. As estratégias podem ser desenvolvidas pelas instituições de saúde, através de suporte on-line de forma a inibir fatores como a insônia, irritabilidade, ansiedade, com intensificação de capacitação e treinamento e contratação de funcionários com diminuição da sobrecarga de trabalho, além da garantia de equipamentos de proteção individual.

\section{REFERÊNCIAS}

BARBOSA, J. D. et al. Fatores de estresse nos profissionais de enfermagem no combate à pandemia da COVID-19: Síntese de Evidências. Com. Ciências da Saúde, v. 31, n. 1, p. 31-47, 2020. Disponível em:

http://www.escs.edu.br/revistaccs/index.php/comunicacaoemcienciasdasaude/article/vie w/651. Acesso em: 12 out. 2020.

BRASIL. Ministério da Saúde. Secretaria de Vigilância em Saúde. Boletim epidemiológico especial doença pelo coronavirus Covid-19. Brasília: Ministério da Saúde, 2020a. Disponível em:

https://antigo.saude.gov.br/images/pdf/2020/July/08/Boletim-epidemiologico-COVID21-corrigido-13h35--002-.pdf. Acesso em: 10 ago. 2020.

BRASIL. Ministério da Saúde. Pesquisa analisa impacto psicologico da covid em profissionais da saúde. Brasília: Ministério da Saúde, 2020b. Disponível em: https://www.gov.br/casacivil/pt-br/assuntos/noticias/2020/julho/saude-mental-pesquisaanalisa-impacto-psicologico-do-enfrentamento-a-covid-19-em-profissionais-da-saude. Acesso em: 18 nov. 2020.

DUARTE, M. Q. et al. Covid-19 e os impactos na saúde mental: uma amostra do Rio Grande do Sul. Ciênc. saúde coletiva, v. 25, n. 9, set. 2020. Disponível em: https://www.scielo.br/j/csc/a/ghSHWNYkP6gqJm4LQVhkB7g/abstract/?lang=pt. Acesso em: 21 out. 2020.

OLIVEIRA, W. A. et al. Impactos psicológicos e ocupacionais das sucessivas ondas recentes de pandemias em profissionais da saúde: revisão integrativa e lições aprendidas. Estudos de Psicologia (Campinas), v. 37, 2020. Disponível em: https://www.scielo.br/j/estpsi/a/ZMN96H6CP5t3MpmyFSrNXPM/?lang=pt. Acesso em: 25 out. 2020. 
OPAS/OMS. Organização Pan-Americana da Saúde. Organização Mundial da Saúde. Folha informativa sobre COVID-19. OPAS/OMS, 2020. Disponível em: https://www.paho.org/pt/covid19. Acesso em: 18 set. 2020.

PRADO, A. D. et al. A saúde mental dos profissionais de saúde frente à pandemia do COVID-19: uma revisão integrativa. Revista Eletrônica Acervo Saúde, v. 46, e4128, 2020. Disponível em: https://acervomais.com.br/index.php/saude/article/view/4128. Acesso em: 19 out. 2020.

SCHMIDT, B. et al. Saúde mental e intervenções psicológicasdiante da pandemia do novo coronavírus (COVID-19). Estudos de Psicologia (Campinas), v. 37, 2020.

Disponível em:

https://www.scielo.br/j/estpsi/a/L6j64vKkynZH9Gc4PtNWQng/?lang=pt. Acesso em: 25 out. 2020.

SHIGEMURA, J. et al. Public responses to the novel 2019 coronavirus (2019-nCoV) in Japan: mental health consequences and target populations. Psychiatry Clin Neurosci., v. 74, n. 4, p. 281-282, 2020. Disponível em:

https://pubmed.ncbi.nlm.nih.gov/32034840/. Acesso em: 18 nov. 2020.

SILVA, A. G. et al. Mental health: why it still matters in the midst of a pandemic. Braz J Psychiatry., v. 42, n. 3, p. 229-31, 2020. Disponível em:

https://www.scielo.br/j/rbp/a/5NkrjxyF9PYRQmYbGXyX5bw/?lang=en. Acesso em: 28 out. 2020.

WANG, C. et al. Immediate psychological responses and associated factors during the initial stage of the 2019 coronavirus disease (COVID-19) epidemic among the general population in china. Int J Environ Res Public Health., v. 17, n. 5, p. 17-29, 2020.

Disponível em: https://pubmed.ncbi.nlm.nih.gov/32155789/. Acesso em: 10 nov. 2020. 